\title{
Diagnostic Value of Dystrophin Immunostaining in the Diagnosis of Duchenne and Becker Muscular Dystrophy Patients
}

\author{
Shinta Andi Sarasati ${ }^{1,4}$, Kristy Iskandar ${ }^{2,5}$, Maria Alethea Septinastiti ${ }^{3}$, Rusdy Ghazali Malueka ${ }^{3,4}$, Ery Kus Dwianingsih ${ }^{1,4 *} \mathbb{D}$ \\ ${ }^{1}$ Department of Anatomical Pathology, Faculty of Medicine, Public Health and Nursing, Universitas Gadjah Mada, Yogyakarta, \\ Indonesia; ${ }^{2}$ Department of Child Health, Faculty of Medicine, Public Health and Nursing, Universitas Gadjah Mada, Yogyakarta, \\ Indonesia; ${ }^{3}$ Department of Neurology, Faculty of Medicine, Public Health and Nursing, Universitas Gadjah Mada, Yogyakarta, \\ Indonesia; ${ }^{4}$ Dr. Sardjito General Hospital, Yogyakarta, Indonesia; ${ }^{5}$ Academic Hospital, Universitas Gadjah Mada, Yogyakarta, \\ Indonesia
}

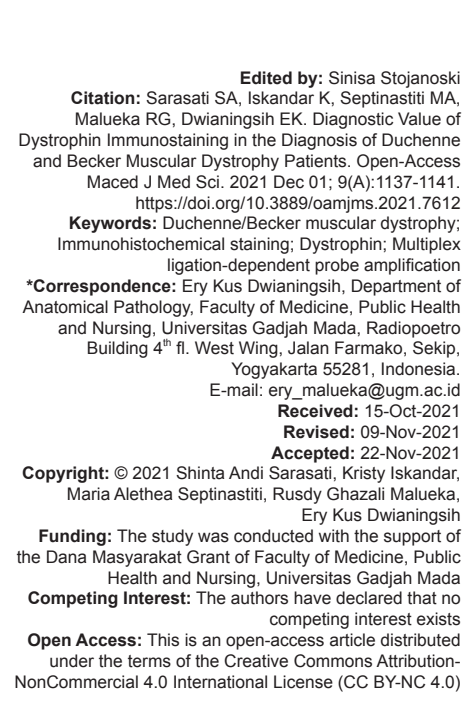

Introduction

Duchenne muscular dystrophy (DMD) is an X-linked recessive muscular disorder caused by the absence or reduction of dystrophin, a cytoskeletal protein. The causative gene, $D M D$, is the largest human gene and is located on chromosome Xp21. The products of $D M D$ provide muscle fiber stability during contractions and maintain membrane stability. Muscle fibers with negative expression of dystrophin are prone to damage since they connect the sarcomeric structure to the extracellular matrix [1]. DMD is characterized by progressive muscular weakness that eventually can progress to respiratory and cardiac failure in some patients which are critical factors that determine their survival [2]. Patients with mutations of the $D M D$ gene may show variable symptoms, ranging from the severe phenotype (DMD) to the milder form, Becker muscular dystrophy (BMD). In DMD, the out-of-frame mutation causes the disruption of the translational reading frame leading to the complete absence of dystrophin. In the milder phenotype, an in-frame mutation still maintains the translational reading frame so that a short but functional dystrophin is able to be produced [3]. When the mutation can maintain the open reading frame, it allows the production of internally deleted, partially functional, dystrophin proteins [4]. DMD and BMD are distinguished by the reading frame hypothesis, because the mutations that cause the distortion of the reading frame may generate a premature termination and loss of dystrophin production, promoting the severe phenotype, DMD [5].

Some methods are available and have been routinely applied to detect deletions and duplications in 
the $D M D$ gene for diagnostic purposes. The multiplex ligation-dependent probe amplification (MLPA) has been a general method to determine the copy number of up to 45 nucleic acid sequences in one single reaction [6]. In the amplification process, multiple pairs of oligonucleotides comprising universal primer sequences, variable lengths of stuffer sequences, and genomic target sequences that hybridize at adjacent positions are ligated with a thermostable ligase enzyme. MLPA has been demonstrated to be a reliable and faster method for quantitative identification of all $D M D$ genes to detect the deletions and duplications [7].

In patients with DMD, the immunohistochemical (IHC) analysis from muscle biopsy specimens shows complete absence of dystrophin, while in BMD, patients may have $10-40 \%$ of the normal protein, partly expressed in the sarcolemma of the muscle fibers [1], [8].

The national health insurance scheme in Indonesia does not cover genetic testing, so muscle biopsy with $\mathrm{IHC}$ staining becomes an important diagnostic tool to diagnose DMD/BMD. The immunostaining of fresh frozen muscle samples is still the standard method to detect the expression of proteins in patients with DMD/BMD using commercially available antibodies [9]. While the snap frozen technique has some advantages, this method needs cryostat and an immunofluorescence microscope to produce fresh frozen sections that are not widely available in all health institutions, especially in developing countries, such as Indonesia. In our institution, formalin-fixed paraffinembedded (FFPE) specimens are more readily available to be used for analysis. However, the successful IHC staining of sarcolemma membrane-associated proteins in FFPE muscle samples has rarely been described. As a diagnostic tool, it is important to establish the accuracy of the method to ensure the diagnostic reliability. The objectives of this study were to report the sensitivity, specificity, positive predictive value (PPV), negative predictive value (NPV), and the accuracy of IHC in diagnosis of Duchenne and Becker muscular dystrophy compared with the MLPA technique as a gold standard.

\section{Materials and Methods}

\section{Samples and data collection}

Twenty-six patients were recruited in the pediatric and neurological departments of Dr. Sardjito and Universitas Gadjah Mada (UGM) Hospital, Yogyakarta, from 2017 to 2018. The oldest was 21 years old and the youngest was 6 years old. Patients who showed progressive muscle weakness, calf hypertrophy, and elevated serum creatine kinase levels were enrolled in this study. Routine histological analysis of muscle biopsies revealed a dystrophic myopathy in all cases and was followed with immunostaining of dystrophin to confirm the protein expression. Written informed consent for genetic examination was obtained from patients' parents. The study protocol was approved by the Medical and Health Research Ethics Committee of the Faculty of Medicine, Public Health and Nursing, UGM (KE/0615/05/2021).

\section{Immunohistochemistry staining}

Muscle biopsy specimens were investigated in our routine diagnostic pathology laboratory. Paraffin blocks were cut in $3 \mu \mathrm{m}$ thickness and placed on charged slides (Superfrost Plus slides; Thermo Scientific (Newcastle upon Tyne, United Kingdom). They were dried at $60^{\circ} \mathrm{C}$ for $30 \mathrm{~min}$, and then dewaxed, rehydrated, and underwent a heat-mediated antigen retrieval method. Sections were pre-treated with Tris-EDTA in $\mathrm{pH} 9.0$ at $95^{\circ} \mathrm{C}$ for 10 min using a pressure cooker, incubated in room temperature for $10 \mathrm{~min}$, and washed with phosphate buffer saline for $30 \mathrm{~min}$. Endogenous peroxidase blocking was done by adding 1-2 drops of $5 \%$ hydrogen peroxidase. After dipping the slide in distilled water, nonspecific background blocking was executed using bond primary antibody diluent (Leica) at room temperature for $30 \mathrm{~min}$. Mouse monoclonal antibody of Dys2 (Novocastra, Leica) was introduced with dilution 1:20, followed with UltraTek Anti-Polyvalent and UltraTek HRP incubation each for $10 \mathrm{~min}$. Immunoreactions were visualized using 3,3'-diaminobenzidine tetrahydrochloride hydrate with subsequent counterstaining of Mayer's hematoxylin. The slides were then dehydrated, cleared, and mounted. Complete loss dystrophin staining resulted in the diagnosis of DMD, while partly loss dystrophin staining revealed BMD as diagnosis.

\section{Genomic DNA extraction}

Each patient provided $3 \mathrm{~mL}$ of EDTA peripheral whole blood sample. Genomic DNA was extracted using Qiagen ${ }^{\circledR}$ QIAamp DNA Mini Kit, according to the manufacturer's protocol. DNA of each sample was purified and $100 \mathrm{ng}$ of it was used for MLPA analysis.

\section{MLPA}

Mutation in DMD gene was detected using MLPA (SALSA MLPA Probemix P034/P035, MRC Holland) according to manufacturer's protocol. All reactions were performed on a thermal cycler PCR Applied Biosystem Veriti 96. One hundred nanograms of sample DNA were diluted in $5 \mu$ l deionized water and denatured at $98^{\circ} \mathrm{C}$ for 15 min before addition of $3 \mu$ MLPA probe mix and buffer. The reaction mixture was denatured at $95^{\circ} \mathrm{C}$ for $1 \mathrm{~min}$ and incubated for $16-18 \mathrm{~h}$ at $60^{\circ} \mathrm{C}$ to secure the specific hybridization of the probes with the target sequences. In ligation step, after the hybridization, ligase mixture $(32 \mu \mathrm{l})$ was added and incubated at 
$54^{\circ} \mathrm{C}$ for 15 min to maintain the ligation reaction. This ligation process was stopped by heating at $98^{\circ} \mathrm{C}$ for $5 \mathrm{~min}$. Ten microliters of this product were mixed with $30 \mu \mathrm{L}$ of PCR buffer and put in a thermocycler at $60^{\circ} \mathrm{C}$. Subsequently, a $10 \mu \mathrm{L}$ reaction mix was added, which contained dNTPs, Taq polymerase, and one unlabeled and one labeled PCR primers that complementary to the universal primer sequences on the MLPA probes. PCR was done for 35 cycles $\left(95^{\circ} \mathrm{C}\right.$ for $30 \mathrm{~s} ; 60^{\circ} \mathrm{C}$ for $30 \mathrm{~s}$, and $70^{\circ} \mathrm{C}$ for $\left.30 \mathrm{~s}\right)$. The fragments were analyzed on an $\mathrm{ABI}$ model 3500 capillary sequencer (Applied Biosystems) with the GeneScan software using GeneScan $600 \mathrm{Liz}$ size standards (Applied Biosystems). Each of the peaks that represent each exon was analyzed based on the difference in migration relative to the size standards and compared to control samples. Deletion and duplication were identified and analyzed for their reading frame status, to determine whether they are in-frame or out-offrame mutations. Patients with MLPA result of no deletion or no duplication were excluded from this study.

\section{Data analysis}

The primary aims of our study were to calculate the sensitivity, specificity, PPV, NPV, and accuracy for IHC staining of dystrophin based on MLPA as the gold standard. To calculate sensitivity and specificity, $2 \times$ 2 tables were generated to analyze IHC results from muscle biopsy (absent or patchy) and genetic results (out-of-frame or in-frame status) from MLPA.

\section{Results}

Among 26 male patients, the mean of patients age was 9 years old, the youngest was 6 years old, while the oldest was 21 years old. Most of the patients (57.7\%) were aged 9 years old or older. The $\mathrm{IHC}$ results in normal muscle were localized at the sarcolemma of the fibers (Figure. 1).

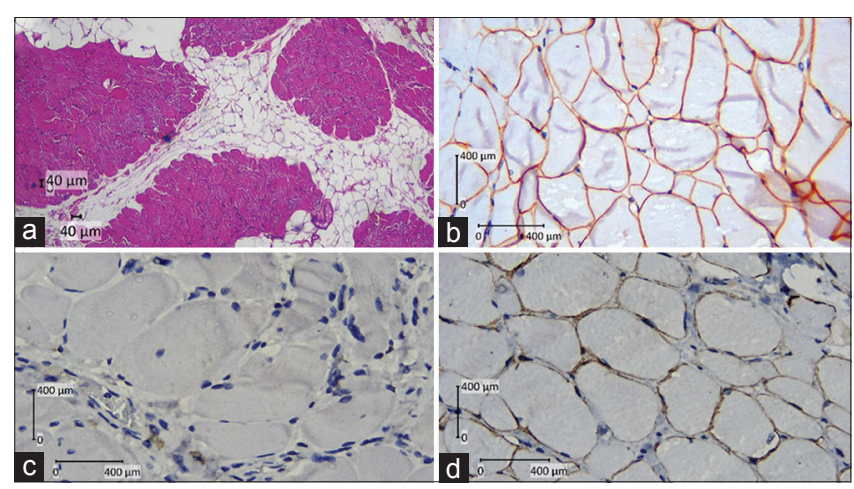

Figure 1: Serial sections of the skeletal muscle biopsy; (a) disruption of muscle fascicles with marked fat proliferation; (b) complete dystrophin expression at sarcolemma membrane in normal muscle (control); (c) negative staining in DMD patient; and (d) patchy staining in BMD patient
Data of all patients are summarized in Table 1. Among the 26 patients with DMD studied, a totally negative immunostaining was observed in most patients, in as many as $20(20 / 26 ; 77 \%)$. In the remaining 6 patients $(6 / 26 ; 23 \%)$, a patchy staining of the sarcolemma was seen in a proportion which was varied from $4 \%$ to $30 \%$ of scattered fibers. In addition, there was no patient with complete dystrophin expression.

Table 1: Immunohistochemistry and MLPA results of 26 male samples

\begin{tabular}{lllll}
\hline No. & Code & IHC & MLPA & $\begin{array}{l}\text { Type of exon } \\
\text { deletion or } \\
\text { duplication }\end{array}$ \\
\hline 1 & & & & \\
2 & DMD-4-MFARH & DMD & No del no dup & \\
3 & DMD-5-MHH & DMD & Out-of-frame & del 53-54 \\
4 & DMD-8-NSA & DMD & Out-of-frame & del 17-43 \\
5 & DMD-10-AP & BMD & Out-of-frame & del 51 \\
6 & DMD-11-NPP & BMD & Out-of-frame & del 46-51 \\
7 & DMD-14-RPP & DMD & No del no dup & \\
8 & DMD-15-AM & DMD & Out-of-frame & del 48-50 \\
9 & DMD-16-AAM & DMD & Out-of-frame & del 45-52 \\
10 & DMD-17-MDZ & DMD & Out-of-frame & del 52 \\
11 & DMD-19-RKS & DMD & Out-of-frame & dup 2-62 \\
12 & DMD-20-PBT & BMD & In-frame & del 7-43 \\
13 & DMD-21-STA & DMD & Out-of-frame & dup 2-62 \\
14 & DMD-27-FWWA & DMD & No del no dup & \\
15 & DMD-28-SKF & DMD & In-frame & del 47 \\
16 & DMD-30-DNM & DMD & out-of-frame & del 51-54 \\
17 & DMD-34-BWCN & DMD & Out-of-frame & del 49-50 \\
18 & DMD-35-DCS & BMD & Out-of-frame & dup 14-17 \\
19 & DMD-36-ANA & DMD & Out-of-frame & del 18-47 \\
20 & DMD-37-NFA & DMD & Out-of-frame & del 56-74 \\
21 & DMD-38-AS & DMD & In-frame & del 45-49 \\
22 & DMD-39-GR & BMD & Out-of-frame & del 18-34 \\
23 & DMD-53-GAP & DMD & Out-of-frame & del 51 \\
24 & DMD-64-MA & DMD & No del no dup & \\
25 & DMD-65-SNR & DMD & Out-of-frame & del 48-50 \\
26 & DMD-66A-PPCA & DMD & Out-of-frame & del 38-43 \\
\hline DMD: Duchen & DMD-67-ML & muscular dystrophy; BMD: Becker muscular dystrophy; del: Deletion; dup: Duplication.
\end{tabular}

MLPA results revealed 18 (18/26; 69.3\%) patients with deletion and $3(3 / 26 ; 11.5 \%)$ patients with duplication. Five $(5 / 26 ; 19.2 \%)$ patients who showed no deletion nor duplication were excluded from the analysis. Among 21 patients with deletion or duplication, $18(18 / 21 ; 85.7 \%)$ patients were out-of-frame (DMD) and $3(3 / 21 ; 14.3 \%)$ patients were in-frame (BMD). Therefore, 21 patients $(21 / 26 ; 80.8 \%)$ were analyzed with a $2 \times 2$ table, as shown in Table 2 .

Table 2: Analysis result dystrophin immunohistochemistry expression and MLPA

\begin{tabular}{llll}
\hline Immunostaining/MLPA & MLPA result & Total \\
\cline { 2 - 3 } & Out-frame & In-frame & \\
\hline Dystrophin immunostaining & $14(66.7)$ & $2(9.5)$ & 16 \\
DMD, $\mathrm{n}(\%)$ (complete loss) & $4(19)$ & $1(4.8)$ & 5 \\
BMD, $\mathrm{n}(\%)$ (partial expression) & $18(85.7)$ & $3(14.3)$ & 21 \\
Total & &
\end{tabular}

The complete absence of dystrophin staining was matched with out-of-frame mutation by MLPA in 14 patients (14/21, 66,7\%). However, 4 patients (4/21; 19.05\%) with out-of-frame mutation showed patchy staining of dystrophin. One patient $(1 / 21 ; 4.76 \%)$ with in-frame mutation also showed patchy expression of dystrophin.

Table 3: Sensitivity, specificity, PPV, NPV, and accuration between immunohistochemistry and MLPA result to detect dystrophin

\begin{tabular}{llllll}
\hline Method & Sensitivities (\%) & Specificities (\%) & PPV (\%) & NPV (\%) & Accuration (\%) \\
\hline IHC & 77.7 & 33.3 & 87.5 & 20 & 71.4 \\
\hline PPV: Positive predictive value, NPV: Negative predictive value. & & &
\end{tabular}


The sensitivity of dystrophin IHC was $77.78 \%$, specificity $33.33 \%$, PPV $87.5 \%$, NPV 20\%, and accuracy $71.43 \%$ (Table 3 ).

\section{Discussion}

The fresh frozen analysis from muscle biopsy is the standard examination for muscular dystrophy, but this service is not widely available in countries with limited facilities to perform immunofluorescence analysis. The successful immunostaining of sarcolemma membraneassociated proteins in a previous study inspired the use of IHC for the diagnosis of common muscular dystrophy. When it is combined with clinical and histopathology findings of the muscle biopsy, especially in cases when frozen muscle sections cannot be obtained, $\mathrm{IHC}$ is beneficial for the diagnosis and screening of DMD/BMD [8].

IHC analysis in dystrophinopathies routinely involves the use of antibodies against C-terminal (Dys2), rod domain (Dys1), and optionally $\mathrm{N}$-terminal (Dys3) of dystrophin protein [10]. In this study, IHC was performed using the Dys-2 antibody since successful immunostaining of Dys-2 using FFPE muscle sections had been reported previously [11], [12]. The Dys2-binding domain is encoded by exons 77-79 of dystrophin in the C-terminal [13]. Since no deletions nor duplications were found in exons 77-79 in this study, the use of Dys-2 antibody alone is sufficient for analysis.

There were six patients with a discrepancy of the IHC and MLPA results with $9.5 \% \quad(2 / 21)$ false positive and $19 \%$ (4/21) false negative. The false-positivity results may occur due to non-immunological binding or protein substrate reaction products, or endogenous enzymes reaction such as pseudoperoxidase, endogenous peroxidase, or endogenous biotin [14], [15]. This study had eliminated endogenous enzymes reactions using Tris-EDTA pre-treatment in $\mathrm{pH} 9.0$ for $10 \mathrm{~min}$. Another reason that may explain false positivity is revertant fibers, which are muscle fibers that express a smaller, but functional, dystrophin protein due to exon skipping and clonally expand in size along with increasing age through the process of muscle degeneration or regeneration [16]. The precise mechanisms by which revertant fibers arise and expand are poorly understood [17].

Common causes of false negativity are poor tissue fixation, over diluted antibody, and poor optimization of the epitope retrieval method [17]. Another reason for the discrepancy is the reading frame theory in BMD, because BMD has higher proportions of duplications, various mutation distribution and higher exceptions to the reading frame rule. Mutations that maintain the reading frame generally result in abnormal but partly functional dystrophin [14]. However, when alternative splicing of the mutant exon restores the reading frame, it appears to be excluded at both the mRNA and protein levels. Some mechanisms that may modulate this discrepancy are ribosomal frameshift, unexpected alternative splicing, exon skipping of mutated exon, and somatic mosaicism [15].

The MLPA technique has increased the mutation pick-up rate [18]. Moreover, the technique also enables the identification of carrier individuals. The MLPA test has proved to be a powerful tool in detecting deletions or duplications in the $D M D$ gene [19]. Studies on comparing IHC staining with genetic mutation using MLPA in patients with DMD have received relatively little investigation.

Sensitivity, specificity, PPV, NPV, and accuration of dystrophin immunostaining were $77.78 \%$, $33.33 \%, 87.5 \%, 20 \%$, and $71.4 \%$, respectively. High sensitivity of dystrophin immunostaining showed that the method was reliable to detect dystrophin protein in tested samples and imply that any positivity of dystrophin expression will exclude the diagnosis of DMD [20]. Although this study showed low specificity, high sensitivity of dystrophin staining can be alternative method to differentiate DMD from BMD, especially in developing country, such as Indonesia, where genetic testing is not covered by insurance while muscle biopsy is. Unlike sensitivity and specificity, PPVs/NPVs are chiefly subjected to disease prevalence in the examined population. Prevalence influences PPV and NPV differently. PPV is increasing, while NPV declines with the increased prevalence of the disease in a population. The change in PPV is more significant, while NPV is only slightly affected by the disease prevalence [21].

A study from India, comparing the sensitivity and the pattern of mutations by both $\mathrm{MPCR}$ and MLPA in the same cohort of DMD, revealed that $36.4 \%$ of MLPAnegative cases were confirmed to be diagnosed with DMD by immunostaining with high accuracy [7]. A comparative study of PCR-based deletion detection and $\mathrm{IHC}$ in Brazil stated that immunostaining can be a gold standard technique to diagnose DMD/BMD in developing countries. However, since muscle biopsy is an invasive procedure, it should only be performed in cases when PCR-based mutation detection using blood is unsuccessful to detect deletions [22].

\section{Conclusion}

Muscle biopsy followed by IHC staining provides diagnostic tools for DMD or BMD with highly sensitivity. The protein-based strategy can be the most efficient way to approach diagnosis of DMD/BMD in health-care centers with limited setting. Immunohistochemistry Dys-2 can be an alternative method to distinguish BMD 
and DMD. The combination of direct dystrophin analysis and genetic testing will give optimum diagnostic and prognostic accuracy.

\section{References}

1. Cohn RD, Campbell KP. Molecular basis of muscular dystrophies. Muscle Nerve. 2000;23(10):1456-71. https:// doi.org/10.1002/1097-4598(200010)23:10<1456:AIDMUS2>3.0.CO;2-T

PMid: 11003781

2. Van Putten M, Hulsker M, Nadarajah VD, van Heiningen $\mathrm{SH}$, van Huizen $\mathrm{E}$, van Iterson $\mathrm{M}$, et al. The effects of low levels of dystrophin on mouse muscle function and pathology. PLoS One. 2012;7(2):e31937. https://doi.org/10.1371/journal. pone.0031937

PMid:22359642

3. Malik V, Rodino Klapac LR, Viollet L, Mendell JR. Aminoglycoside-induced mutation suppression (stop codon readthrough) as a therapeutic strategy for Duchenne muscular dystrophy. Ther Adv Neurol Disord. 2010;3(6):379-89. https:// doi.org/10.1177/1756285610388693

PMid:21179598

4. Bladen CL, Salgado D, Monges S, Foncuberta ME, Kekou K, Kosma $\mathrm{K}$, et al. The TREAT-NMD DMD global database: Analysis of more than 7,000 duchenne muscular dystrophy mutations. Hum Mutat. 2015;36(4):395-402. https://doi. org/10.1002/humu.22758

\section{PMid:25604253}

5. Nadifi S, Bellayou H, Hamzi K, Rafai MA, Karkouri M, Slassi I, et al. Duchenne and Becker muscular dystrophy: Contribution of a molecular and immunohistochemical analysis in diagnosis in Morocco. J Biomed Biotechnol. 2009;2009:325210. https://doi. org/10.1155/2009/325210

\section{PMid:19461958}

6. Janssen B, Hartmann C, Scholz V, Jauch A, Zschocke J. MLPA analysis for the detection of deletions, duplications and complex rearrangements in the dystrophin gene: Potential and pitfalls. Neurogenetics. 2005;6(1):29-35. https://doi.org/10.1007/ s10048-004-0204-1

PMid:15655674

7. Manjunath $M$, Kiran $P$, Preethish-Kumar $V$, Nalini A, Singh $R$, Gayathri N. A comparative study of mPCR, MLPA, and muscle biopsy results in a cohort of children with Duchenne muscular dystrophy: A first study. Neurol India. 2015;63(1):58-62. https:// doi.org/10.4103/0028-3886.152635

PMid:25751470

8. Suriyonplengsaeng C, Dejthevaporn C, Khongkhatithum C, Sanpapant S, Tubthong N, Pinpradap K, et al. Immunohistochemistry of sarcolemmal membrane-associated proteins in formalin-fixed and paraffin-embedded skeletal muscle tissue: A promising tool for the diagnostic evaluation of common muscular dystrophies. Diagn Pathol. 2017;12(1):1-10. https://doi.org/10.1186/s13000-017-0610-y

PMid:28219397

9. Bushby K, Finkel R, Birnkrant DJ, Case LE, Clemens PR, Cripe $\mathrm{L}$, et al. Diagnosis and management of Duchenne muscular dystrophy, Part 1: Diagnosis, and pharmacological and psychosocial management. Lancet Neurol. 2010;9(1):77-93. https://doi.org/10.1016/S1474-4422(09)70271-6

PMid:19945913
10. Vogel H, Zamecnik J. Diagnostic immunohistology of muscle diseases. J Neuropathol Exp Neurol. 2005;64(3):181-93. https:// doi.org/10.1093/jnen/64.3.181

PMid: 15804049

11. Hoshino S, Ohkoshi N, Watanabe M, Shoji $S$ Immunohistochemical staining of dystrophin on formalin-fixed paraffin-embedded sections in Duchenne/Becker muscular dystrophy and manifesting carriers of Duchenne muscular dystrophy. Neuromuscul Disord. 2000;10(6):425-9. https://doi. org/10.1016/S0960-8966(99)00116-9

PMid:10899449

12. Sheriffs IN, Rampling D, Smith VV. Paraffin wax embedded muscle is suitable for the diagnosis of muscular dystrophy. J Clin Pathol. 2001;54(7):517-20. http://doi.org/10.1136/jcp.54.7.517 PMid:11429422

13. Nishida A, Kataoka N, Takeshima Y, Yagi M, Awano H, Ota M et al. Chemical treatment enhances skipping of a mutated exon in the dystrophin gene. Nat Commun. 2011;2(1):308. https://doi. org/10.1038/ncomms1306

14. Gibbs EM, Barthélémy F, Douine ED, Hardiman N, Shieh PB Khanlou N, et al. Large in-frame 5' deletions in DMD associated with mild Duchenne muscular dystrophy: Two case reports and a review of the literature. Neuromuscul Disord. 2019;29(11):863-73. https://doi.org/10.1016/j.nmd.2019.09.009 PMid:31672265

15. Nadkarni J, Dastur R, Gaitonde P, Khadilkar S. Becker muscular dystrophy in Indian patients: Analysis of dystrophin gene deletion patterns. Neurol India. 2008;56(3):374. https://doi. org/10.4103/0028-3886.40961

\section{PMid: 18974567}

16. Nowak KJ, Davies KE. Duchenne muscular dystrophy and dystrophin: Pathogenesis and opportunities for treatment. EMBO Rep. 2004;5(9):872-6. https://doi.org/10.1038/ sj.embor.7400221

PMid:15470384

17. Echigoya $\mathrm{Y}$, Lee J, Rodrigues $\mathrm{M}$, Nagata $\mathrm{T}$, Tanihata J, Nozohourmehrabad A, et al. Mutation types and aging differently affect revertant fiber expansion in dystrophic Mdx and Mdx52 mice. PLoS One. 2013;8(7):e69194. https://doi.org/10.1371/ journal.pone.0069194 PMid:23894429

18. Schwartz M, Dunø M. Improved molecular diagnosis of dystrophin gene mutations using the multiplex ligation-dependent probe amplification method.Genetic Testing. 2004;8(4):361-7. https:// doi.org/10.1089/gte.2004.8.361

PMid: 15684864

19. Todorova A, Todorov T, Georgieva B, Lukova M, Guergueltcheva V, Kremensky I, et al. MLPA analysis/ complete sequencing of the DMD gene in a group of Bulgarian Duchenne/Becker muscular dystrophy patients. Neuromuscul Disord. 2008;18(8):667-70. https://doi.org/10.1016/j. nmd.2008.06.369

20. Grunau G, Linn S. Commentary: Sensitivity, specificity, and predictive values: Foundations, pliabilities, and pitfalls in research and practice. Front Public Health. 2018:6:1-4. https:// doi.org/10.3389/fpubh.2018.00256

21. Wong HB, Lim GH. Measures of diagnostic accuracy: Sensitivity, specificity, PPV and NPV. Proc Singapore Healthc. 2011;20(4):316-8.

22. Werneck LC, Scola RH, Henrique G, Maegawa B. Comparative analysis of PCR-deletion detection and immunohistochemistry in Brazilian Duchenne and Becker muscular dystrophy patients. Am J Med Genet. 2001;103(2):115-20. https://doi.org/10.1002/ ajmg.1508

PMid:11568916 